\title{
DIVERTÍCULO DE ZENKER Femenina 54 años
}

Denisse Rodríguez Mena ${ }^{1}$ José Alberto Ayi Wong ${ }^{2}$

${ }^{1}$ Médico General, Universidad de Costa Rica. San José, Costa Rica. Correspondencia: demirod@hotmail.com.

${ }^{2}$ Especialista en Cirugía General, Asistente del Servicio Emergencias Quirúrgicas. Hospital San Juan de Dios. San José, Costa Rica.

\section{RESUMEN}

El divertículo de Zenker es una entidad poco frecuente. Se presenta predominantemente en pacientes masculinos, y tiene como síntomas principales: disfagia, regurgitación alimentaria, halitosis, broncoaspiración y sepsis respiratorias. $\mathrm{Su}$ localización es por lo general del lado izquierdo del esófago y su tratamiento definitivo es quirúrgico.

Se presenta el caso de una paciente femenina, joven, con sintomatología esofágica, quien se diagnostica con divertículo de Zenker, de localización posterior, a la cual se le realiza diverticulectomía con miomectomía como tratamiento quirúrgico, con resolución completa de los síntomas y excelente recuperación.

\section{PALABRAS CLAVE}

Divertículo de Zenker, miomectomía, divertículo esofágico, divertículo faringoesofágico.

\footnotetext{
ABSTRACT

Zenker's diverticulum is a rare condition, that has a male predominancy. The most frequent symptoms are dysphagia, food regurgitation, halitosis, aspiration and pulmonary sepsis. It is usually located on the left side of the esophagus and the treatment is definitively surgical.

We present the case of a young female patient, with esophageal symptoms, who is diagnosed with Zenker's diverticulum, in a posterior location, who was surgically treated with a diverticulectomy and myomectomy, with
}

complete resolution of symptoms and excellent recovery.

\section{KEY WORDS}

Zenker's diverticulum, myomectomy, esophageal diverticulum, pharyngoesophageal diverticulum.

\section{CASO CLÍNICO}

Paciente femenina, 54 años, vecina de Goicoechea, conocida sana, no tabaquista ni etilista. Con historia de dos años de evolución de disfagia, espasmos laríngeos, tos, disfonía, ronquera, sensación de masa, faringitis a repetición, síntomas que han ido en aumento en los últimos seis meses. No presentó halitosis ni reflujo gastroesofágico.

Valorada inicialmente en el Servicio de Otorrinolaringología de un hospital central, donde no se le encontró ninguna alteración al examen físico.

Debido a la persistencia de los síntomas se le realiza un esofagograma con medio de contraste baritado, bajo control fluoroscópico, en el que se observa a nivel de esófago superior $\left(5^{\circ}\right.$ vértebra cervical) una saculación extraluminal posterior, con medidas de $11,17 \mathrm{~mm}$. (AP, L). La pared presentaba contornos lisos y un patrón de pliegues finos fundamentalmente longitudinales. El paso esofágico se daba sin dificultad y sin indicios de hernia hiatal ni reflujo. Cuello y tórax sin otras patologías. 
La esofagogastroduodenoscopía evidenció un divertículo posterior en esófago cervical, con cambios de gastritis crónica. El resto de exámenes, tanto hemograma completo, radiografía de tórax y electrocardiograma, resultaron completamente normales.

Se intervino quirúrgicamente por el Servicio de Cirugía de Tórax. El abordaje fue mediante una cervicotomía izquierda y se documentó la presencia de un divertículo de Zenker de aproximadamente $1,5 \mathrm{~cm}$ de diámetro en pared posterior, sin otras alteraciones. Se realiza resección y miotomía del músculo cricofaríngeo y del músculo constrictor inferior. Se le reinició la vía oral al tercer día postoperatorio, sin complicaciones.

\section{DISCUSIÓN Y ANÁLISIS DEL CASO}

El divertículo faringoesofágico fue descrito inicialmente por el cirujano inglés Abraham Ludlow, en 1769, pero fue el cirujano alemán Albert von Zenker quien en 1874 publicó una revisión de 22 casos y se le otorgó su nombre. ${ }^{(1,2,3)}$

La localización del divertículo la hizo Gustav Killian en 1907, quien lo ubicó entre las fibras del músculo constrictor inferior de la faringe y las del cricofaríngeo, y a esta zona se le llamó "Triángulo de Killian". ${ }^{(1,4)}$ El primero en realizar la extirpación del divertículo fue William Wheeler en $1886 .{ }^{(1)}$

Es una patología infrecuente, ${ }^{(5)}$ con una prevalencia en la población del $0.01-0.11 \%{ }^{(4)} \mathrm{Se}$ presenta más en el sexo masculino, ${ }^{(6)}$ con una relación $2: 1^{(7)}$ y representa el $60-65 \%$ de todos los divertículos de esófago. ${ }^{(1)}$

Por lo general este divertículo se presenta en personas mayores, hombres generalmente de más de 60 años. ${ }^{(1,3,4)}$

Existen varias teorías sobre su posible origen, pues aún no existe una etiología clara. Una teoría es que el reflujo gastroesofágico puede generar espasmo cricofaríngeo y acalasia cricofaríngea. Otra teoría propuesta es la producida por espasmos del esfínter esofágico superior. ${ }^{(1)} \mathrm{Se}$ han descrito dos factores necesarios para que se produzca este divertículo, uno es la incoordinación motora entre el músculo constrictor inferior de la faringe y el músculo cricofaríngeo, y su asociación con un defecto anatómico entre ellos. ${ }^{(1)}$

Se genera una incoordinación al momento de la deglución, por lo que aumenta la presión y provoca la formación del saco que irá aumentando de tamaño con el paso de meses o años. ${ }^{(6)}$ Se pueden clasificar como divertículos por tracción o por pulsión. Los que se producen por pulsión están recubiertos de mucosa solamente, mientras que los que se generan por tracción poseen todas las capas del esófago. ${ }^{(7)}$

La disfunción de los músculos cricofaríngeos, crean una zona de alta presión en la hipofaringe formándose el saco en la zona de debilidad que se denomina triángulo de Killian. ${ }^{(8)}$

También se ha sugerido la existencia de una relación genética. Esto apoyado por el reporte de familias con transmisión de esta patología, y la evidencia de que gemelos idénticos la presentan. ${ }^{(9)}$ Otras patologías relacionadas son los distintos trastornos neurogénicos, y el antecedente de cirugía espinal cervical anterior. $^{(8,9)}$

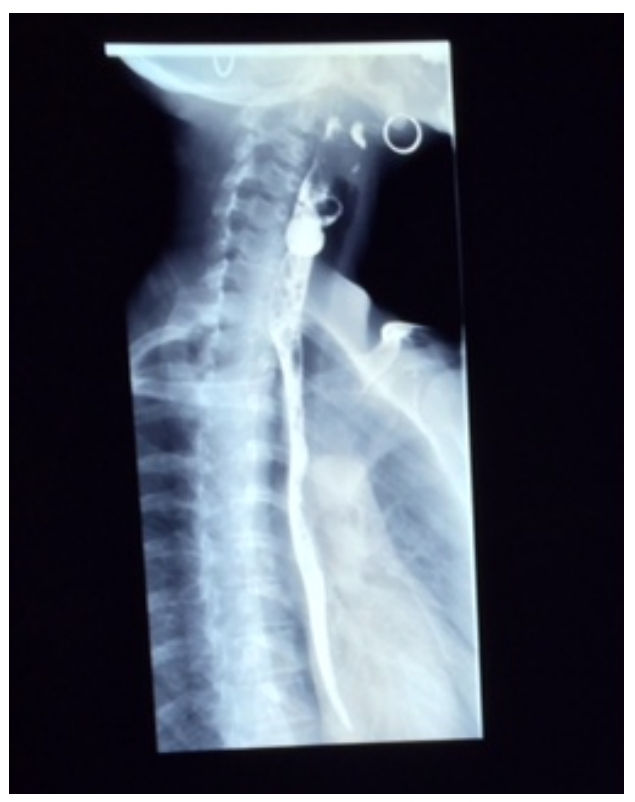

Figura 1. Esofagograma realizado que revela el divertículo localizado en esófago superior.

El divertículo se localiza más comúnmente a nivel de C5-C6, usualmente al lado izquierdo del esófago. ${ }^{(5)}$

Los síntomas más frecuentes son: disfagia, regurgitación de alimentos, halitosis, odinofagia, ${ }^{(8)}$ dolor de cuello, tos crónica, ${ }^{(1)}$ 
broncoaspiración, neumonía ${ }^{(4)}$ y sensación de masa en el cuello. ${ }^{(7)}$

En casos extremos esta patología puede ser progresiva y llevar a la afagia total, ${ }^{(3)}$ lo que conlleva a pérdida de peso y desnutrición. ${ }^{(6)}$ Como complicaciones se pueden presentar infección del saco, ulceración, hemorragia, compresión esofágica, arritmia cardiaca, neumonitis supurativa, perforación de la vía aérea $\mathrm{o}$ del mediastino, $\mathrm{y}$ malignización a carcinoma de células escamosas. ${ }^{(5,7)}$

El carcinoma tiene una incidencia de $0.4-1.5 \%{ }^{(4)}$ Los síntomas que pueden sugerir la presencia de carcinoma son: aumento súbito de disfagia, dolor local, hemoptisis o hematemesis.

Al examen físico se puede palpar una masa, borborigmos o crépitos cervicales. ${ }^{(4)}$

El diagnóstico es mediante la confirmación radiológica con estudios con medio de contraste. $^{(6)}$

Se puede realizar la radiografía convencional para valorar el esófago, pero el esofagograma con bario demuestra el tamaño y la forma del saco. $^{(8)}$

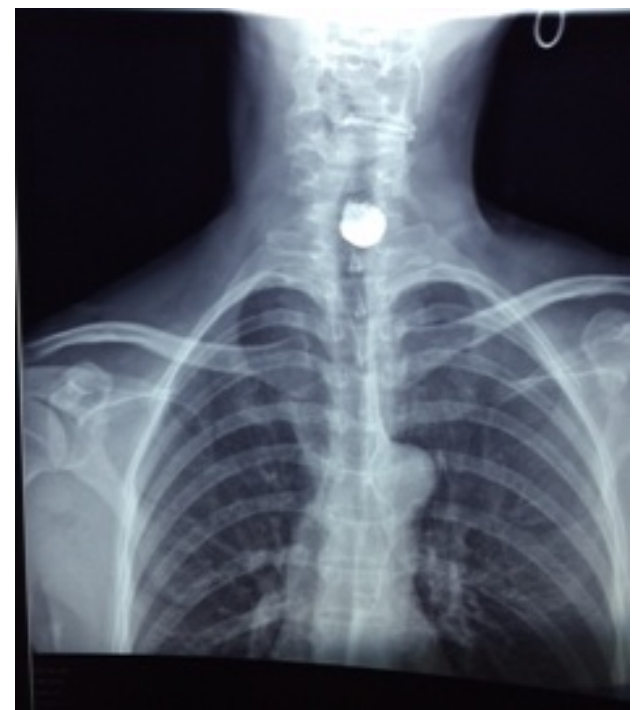

Figura 2. Radiografía de la paciente con imagen del saco diverticular formado hacia posterior.

Se debe realizar endoscopía para tener una vista directa pero no se debe realizar como abordaje inicial. $^{(8)}$

En el caso en el que el divertículo sea sintomático, el tratamiento es exclusivamente quirúrgico, ${ }^{(2)}$ sin embargo se deben valorar las posibles complicaciones de esta cirugía ya que la mayoría de los pacientes son de edad avanzada. ${ }^{(10)}$

El abordaje es mediante cervicotomía sobre el borde anterior del músculo esternocleidomastoideo, y realizar diverticulectomía con miotomía cricofaríngea para evitar recidivas. ${ }^{(3,7)}$

Como alternativa para pacientes con alto riesgo quirúrgico se puede realizar el tratamiento por medio de endoscopia flexible, con resultados exitosos. $^{(8)}$

Las complicaciones postoperatorias son principalmente dolor torácico, persistencia del divertículo, mediastinitis, fístula ${ }^{(11)}$ o estenosis esofágica. ${ }^{(2)}$ Estas complicaciones se ven más comúnmente con la endoscopia. ${ }^{(12)}$

\section{BIBLIOGRAFÍA}

1. Pérez, E; Pérez, J; Sahagún, F; Abdo, J; Murguía, D. Diagnóstico y tratamiento del divertículo de Zenker. Revista Médica del Hospital General de México, 2005; 68:3, 155-159.

2. Mainieri, J; Calderón, M; Chamorro, R; Mainieri, G. Divertículo faringe-esofágico. Acta Médica Costarricense, 2006; 48:4, 215 218.

3. Valdes, M; Álvarez, T; Quintero, L; Cabrera, C; Amurrio, R. Diverticulo de Zenker, presentación de un caso. Gaceta Médica Espirituana, 2008; 10:1, 1-5.

4. Ferreira, L; Simmons, D; Baron, T. Zenker's diverticula: pathophysiology, clinical presentation, and flexible endoscopic management. Diseases Of The Esophagus: Official Journal Of The International Society For Diseases Of The Esophagus, 2008; 21:1, 1-8.

5. Amerasekera, S. Zenkers diverticulum. British Journal Of Hospital Medicine, 2009; 70:1,47.

6. Mayo Clinic. Zenker's diverticulum. A pouch in the throat. Mayo Clinic Health Letter (English Ed.), 2014; 32:4, 6.

7. García, M; Figueredo, P. Zenker diverticuli. Report of two cases. Cirugia y Cirujanos, 2006; 74:4, 283-285.

8. Tian, H; Yuan, W; Johnson, J; Chen, H; Chen, D. Pharyngoesophageal diverticulum: 
a delayed complication of anterior cervical spine surgery. European Spine Journal: Official Publication Of The European Spine Society, The European Spinal Deformity Society, And The European Section Of The Cervical Spine Research Society, 2011; 20, 211-216.

9. Schindler, A; Mozzanica, F; Alfonsi, E; et al. Upper esophageal sphincter dysfunction: diverticula-globus pharyngeus. Annals Of The New York Academy Of Sciences, 2013; 1300:1, 250-260.

10. Giovanni, A; Santini, L. Tratamiento quirúrgico del divertículo faringoesofágico. EMC Cirugía Otorrinolaringóloga y Cervicofacial, 2012; 13:1, 1-8.

11. Keck, T; Rozsasi, A; Grün, P. Surgical treatment of hypopharyngeal diverticulum (Zenker's diverticulum). European Archives Of Oto-Rhino-Laryngology: Official Journal Of The European Federation Of Oto-RhinoLaryngological Societies (EUFOS): Affiliated With The German Society For Oto-Rhino-Laryngology - Head And Neck Surgery, 2010; 267:4, 587-592.

12. van Overbeek, J. Pathogenesis and methods of treatment of Zenker's diverticulum. The Annals Of Otology, Rhinology, And Laryngology, 2003; 112:7, 583-593.

\section{AGRADECIMIENTOS}

Al Servicio de Cirugía Torácica, Hospital Calderón Guardia y al Servicio Cirugía Torácica Hospital San Juan de Dios, San José, Costa Rica. 